\section{O conceito de saúde: ponto-cego da epidemiologia? *}

\section{The concept of health: blind-spot for epidemiology?}

\section{Naomar de Almeida Filho}

Instituto de Saúde Coletiva/Universidade Federal da Bahia

Rua Padre Feijó, 29 - Canela

40110-170 Salvador, BA.

E-mail: naomar@ufba.br

\section{Resumo}

O trabalho faz uma avaliação da proposição de que o conceito de 'saúde' constitui um ponto-cego para a ciência epidemiológica, postulando que não há base lógica para uma definição negativa da Saúde, tanto no nível individual quanto no coletivo. Também analisa brevemente as tentativas de produzir uma "epidemiologia da saúde" em bases simétricas à epidemiologia dos riscos, bem como as abordagens econométricas que reforçam e complementam o repertório epidemiológico destinado à medida da saúde. Focaliza em mais detalhe a abordagem denominada DALY, considerada como protótipo da nova geração de indicadores de saúde, face à sua atualidade $\mathrm{e}$ crescente importância na definição de políticas de financiamento em saúde. Finalmente, constatando o fracasso das propostas metodológicas de avaliação direta dos níveis coletivos de saúde através de indicadores unificados, conclui com uma avaliação das perspectivas atuais da Epidemiologia no sentido da incorporação do objeto complexo da saúde na sua pauta teórica e metodológica.

Palavras-chave: Conceito de Saúde. Epidemiologia. Teoria epidemiológica. Indicadores de saúde. DALY. QALY.

*Anotações para conferência proferida em Concurso para Professor Titular de Epidemiologia do Instituto de Saúde Coletiva da Universidade Federal da Bahia, 31 de julho de 1999. Agradeço a Maurício Barreto, Eduardo Mota e Jairnilson Paim por leituras críticas de diferentes versões do texto. Denise Coutinho revisou estilo e redação 


\section{Abstract}

In this paper, the author evaluates the proposition that the concept of Health is a blind-spot for epidemiologic science, arguing that there is no logical basis for a negative definition of Health, neither at the individual level nor at the collective level. He also briefly analyzes the attempts of producing an epidemiology of health symmetric to the epidemiology of risks, as well as the econometric approaches that reinforce and complement the epidemiologic repertoire for the measurement of health. Due to its growing importance in the definition of budgetary policies in health, the author focuses in more detail on the DALY approach, considered as a prototype of the new generation of health indicators. Finally, recognizing the failure of methodological proposals for a direct evaluation of levels of collective health through unified indicators, the author concludes with an evaluation of the current prospects for Epidemiology in order to integrate the complex object of Health in its theoretical and methodological agenda.

Keywords: Health concept. Epidemiology. Epidemiological theory. Health indicators. DALY. QALY.

\section{Introdução}

Quando a Epidemiologia era apenas uma das ciências básicas da Medicina Preventiva, a vida parecia simples e fácil. Havia a segurança da doença e da causalidade, da bioestatística e do conceito de risco, da terapêutica precoce e da profilaxia. Os objetos de conhecimento e de intervenção apresentavam-se como alvos grandes e lentos, escandalosamente visíveis, definidos com precisão, complacentes, esperando a ação preventiva que, sabia-se, mais cedo ou mais tarde a ciência epidemiológica validaria pelo conhecimento dos ciclos evolutivos das doenças, suas causas e fatores de risco. Dela se exigia apenas a produção de um saber complementar ao saber clínico, capaz de proteger os corpos, as populações e os ambientes dos vetores, dos agentes mórbidos e dos fatores de risco, erradicando-os ou controlando-os, prevenindo assim a ocorrência de doenças.

Em 1946, talvez buscando uma terapêutica para o zeitgeist depressivo do pós-guerra, a Organização Mundial da Saúde reinventou o Nirvana e chamou-o de 'saúde'.

Saúde é o estado de completo bem-estar físico, mental e social, e não meramente a ausência de doença ou incapacidade. ${ }^{2}$

À primeira vista, poderíamos não dar muita atenção à intrigante consigna que teve o mérito (duvidoso) de alimentar, em todo o mundo que se crê civilizado, um novo misticismo sanitário. Porém não creio que se possa fingir indiferença perante o poder simbólico das ideologias, principalmente quando elas catalizam tanto desejo e energia e criam burocracias tão poderosas quanto eficientes no que se refere à própria reprodução. Assim, os competentes burocratas rapidamente se mobilizaram para saber do que se tratava e como se poderia obter aquele "todo completo" sobre o qual somente se informava que não é o "nada da doença".

Certamente como efeito desse processo, no contexto sanitário dos países industrializados, emergiu um movimento ideológico, com características ecumênicas, 
denominado Promoção da Saúde $\cdot^{3-5}$ Promoção, os sanitaristas de carreira, melhor que ninguém, sempre souberam do que se trata. Definir o que é saúde, entretanto, parecia bastante mais difícil. Os novos evangelistas então convocaram publicitários, artistas gráficos, gurus e até mesmo alguns pesquisadores, que contribuíram com logotipos, esquemas e desenhos interessantes, ampliando cada vez mais o "todo completo", incluindo novas modalidades de bem-estar, sempre a garantir que isso nada tem a ver com doença. Produziram-se truísmos e tautologias das mais variadas formas, como por exemplo o caso extremo apresentado na Figura, onde Saúde aparece como uma mandala totalizante das virtudes e valores humanos.

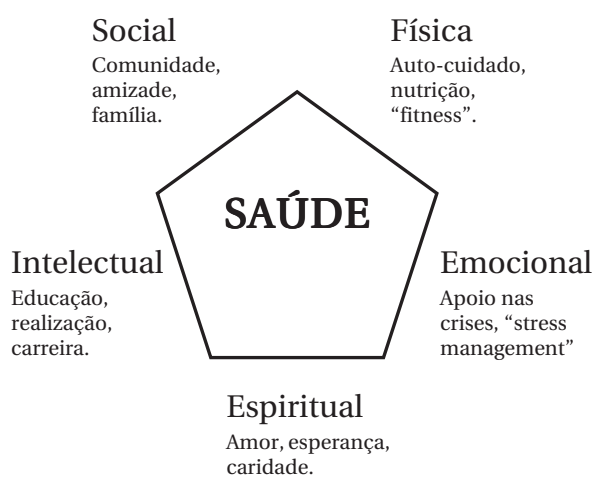

Figura - Modelo-ideal de Saúde. Figure - Ideal-model of health.

Fonte:adaptado de Editorial. Am J Health Promotion 1989;3:3-5. Source: adapted from Editorial. Am J Health Promotion 1989;3:3-5.

Para piorar (ou melhorar, depende do ponto de vista), já faz algum tempo que prometeram saúde para todos... no ano 2000. A OMS escolheu um lugar remoto, bem espiritualizado, chamado Alma Ata, para fazer o lançamento da campanha pela atenção primária à saúde, estratégia que supostamente cumpriria a promessa de universalização da saúde. ${ }^{6}$ Países pós-modernos como Canadá, ${ }^{7}$ Holanda ${ }^{8}$ e Brasil ${ }^{9}$ se engajaram com entusiasmo, terminando por decretar, nas respectivas constituições, que a saúde é direito de todos e dever do Estado.

Resultado: hordas de sanitaristas, expreventivistas, funcionários internacionais, nacionais, regionais e locais, planejadores e gestores, ex-seminaristas, candidatos aos cargos dos funcionários internacionais, nacionais, regionais e locais, militantes, ativistas, enganados e desenganados, iludidos e desiludidos, todos querem saber o que é saúde. A sociedade literalmente bate à porta das instituições acadêmicas e científicas que supostamente deveriam saber o que é, como se mede e como se promove essa tal de "saúde". Traduzindo em linguagem apropriada, isso significa uma tremenda demanda epistemológica resultante de uma ampla pressão social, política, institucional e ideológica. Para atendê-la de modo sério e responsável, o saber preventivista sobre as causas das doenças, que insiste em manter-se médico-clínico em forma e conteúdo, pouco teria a contribuir.

Mas aí surge um grande problema, provocado por essa justa demanda social: por causa do seu subdesenvolvimento epistemológico e conceitual, a Epidemiologia não tem sido capaz de produzir uma referência teórica eficaz sobre o objeto saúde. Minha hipótese é que isso ocorre simplesmente porque o conceito de 'saúde' constitui um dos pontos cegos paradigmáticos da ciência epidemiológica. Em outras oportunidades, ${ }^{10-11}$ tenho assinalado que, além das anomalias kuhnianas, os paradigmas científicos também apresentariam pontos cegos como signos de crise. Refiro-me a problemas ou questões que os próprios paradigmas, consubstanciados pelos agentes históricos engajados na prática institucional da ciência, não permitem "ver" ou sequer toleram que sejam vistos.

É certo que esta lacuna não é exclusiva da Epidemiologia. Em todas as disciplinas que constituem o chamado campo da saúde, noto um flagrante desinteresse em construir conceitualmente o objeto Saúde. Em contraste, pode-se facilmente constatar uma razoável concentração de esforços no sentido de produzir modelos biomédicos de patologia, ${ }^{12-20}$ com forte inspiração mecanicista ou, no máximo, sistêmica que, ao enfatizar os níveis de análise individual e subindividual, terminam por reduzir o alcan- 
ce das suas contribuições. Abordagens dessa ordem mostram-se incapazes de fazer justiça à complexidade dos processos concretos relativos à vida, saúde, aflição, sofrimento, dor, doença, cuidado, cura e morte que ocorrem em agregados humanos históricos.

Em Epidemiologia Sem Números, ${ }^{21}$ anotei que a ciência epidemiológica não portava qualquer teoria da doença, sendo nesse aspecto subsidiária da Clínica. Não deve causar surpresa, portanto, a ausência de uma "teoria científica da saúde" no campo da Epidemiologia. O objeto epidemiológico tem sido construído obedecendo a uma lógica conjuntista, pseudo-probabilística, monótona, que não faz justiça à riqueza e complexidade dos fenômenos da saúde. Essa estratégia de construção teórica baseia-se em uma hermenêutica fixa, matriz de noções já superadas em outros campos científicos de maior maturidade epistemológica, como por exemplo a doutrina do causalismo restrito. ${ }^{10}$ Daí resulta um objeto denominado saúde que de fato refere-se a "doença coletiva", ainda assim tratado de uma maneira parcial e residual como "o risco e seus fatores".

Penso que nem mesmo a coletivização da doença através do conceito de morbidade consegue indiciar "essa coisa chamada saúde". Não é por acaso que os textos epidemiológicos sobre a saúde mostram-se sinuosos e inconvincentes; os seus formuladores patinam sobre metáforas, inventam maneiras indiretas de falar sobre saúde, porém o seu objeto continua sendo a enfermidade e a morte.

No presente trabalho, pretendo analisar a proposição de que o conceito de 'saúde' constitui um ponto-cego para a ciência epidemiológica. Buscarei inicialmente avaliar as limitações da abordagem epidemiológica convencional, dependente de uma definição clínica de doença, para a construção do objeto Saúde. Em segundo lugar, discutirei brevemente as tentativas de produzir uma "epidemiologia da saúde" em bases simétricas à epidemiologia dos riscos. Em terceiro lugar, analisarei criticamente as recentes formulações de viés econométrico que pretensamente reforçam e complementam o repertório epidemiológico destinado à medida da saúde. Como ilustração, focalizarei especialmente a abordagem denominada DALY, patrocinada pelo Banco Mundial e pela OMS, considerada como protótipo da nova geração de indicadores de saúde, face à sua atualidade e crescente importância na definição de políticas de financiamento em saúde. Finalmente, concluo com uma avaliação das perspectivas atuais da Epidemiologia no sentido da incorporação do objeto complexo da saúde na sua pauta teórica e metodológica.

\section{O impasse da clínica da saúde}

Pelos motivos que passo a expor nesta seção, considero que não há qualquer base lógica para uma definição negativa da Saúde, tanto no nível individual quanto no coletivo, mesmo em suas versões aparentemente mais avançadas e completas. Analisemos essa questão primeiro em relação ao nível individual, o que mais uma vez nos leva a revisitar as relações entre a Clínica e a Epidemiologia.

Com vistas a uma formalização preliminar da Saúde no nível individual, devemos considerar as seguintes proposições:

(a) "Nem todos os sujeitos sadios achamse isentos de doença."

(b) "Nem todos os isentos de doença são sadios."

Na prática clínica e na vida cotidiana, identificamos com freqüência indivíduos ativos, social e profissionalmente produtivos, sem sinais de comprometimento, limitação funcional ou sofrimento, auto e hetero-reconhecidos como sadios, que no entanto são portadores de doenças ou sofrem de agravos, seqüelas e incapacidades parciais, mostrando-se muitas vezes profusamente sintomáticos. Outros, ao contrário, encontram-se infectados, apresentam comprometimentos, incapacidades, limitações e sofrimentos sem qualquer evidência clínica de doença. Além da mera presença ou ausência de patologia ou lesão, precisamos também considerar a questão do grau de 
severidade das doenças e complicações resultantes, com repercussões sobre a qualidade de vida dos sujeitos.

Em uma perspectiva rigorosamente clínica, portanto, a Saúde não é o oposto lógico da doença e, por isso, não poderá de modo algum ser definida como "ausência de doença”. Como corolário, tem-se que os estados individuais de saúde não são excludentes vis à vis a ocorrência de doença. Creio que nesse aspecto podemos mesmo parafrasear Caetano Veloso, constatando que "de perto ninguém é sadio". Ou ainda, recorrendo a Canguilhem, ${ }^{22}$ devemos admitir que o oposto lógico da patologia não será de modo algum a Saúde.

Partamos do princípio de que a Saúde pode ser tomada como um atributo individual e, como tal, vulnerável a processos de mensuração. Seria necessário, então, identificar os elementos constitutivos e daí os sinais e sintomas da "síndrome saúde", a fim de verificar a presença, ausência, nível ou grau de pertinência dos indivíduos perante um construto empírico definido de modo sistemático e estável. ${ }^{23}$ Trata-se evidentemente de uma proposta de tratamento simétrico do problema geral da identificação de casos de doença na pesquisa epidemiológica convencional, com a ressalva de que os sinais e sintomas de "saúde" não podem, nesse caso, expressar mera ausência de doença.

Derivadas inicialmente da definição original da OMS, as primeiras tentativas para tratar empiricamente essa questão tomaram ao pé-da-letra que a "saúde" seria composta por três dimensões de bem-estar: físico, mental, social. ${ }^{24-25}$ Como já dispunham de alguns questionários de detecção de casos em inquéritos de morbidade psiquiátrica, concentraram-se no aperfeiçoamento destes e na criação de instrumentos capazes de medir a capacidade física e o bemestar social. No primeiro caso, buscou-se recuperar os conceitos de comprometimento, limitação, incapacidade e desvantagem, agora revestidos de uma certa positividade sob a forma de função, habilidade, capacidade e desempenho. ${ }^{26}$ No se- gundo caso, a teoria do suporte social ${ }^{27}$ passou a ser considerada como um recurso chave para a medida da chamada "saúde social" através dos seus componentes principais: interações interpessoais e participação social. Formulações subseqüentes ${ }^{28}$ subdividiram ou reestruturaram as dimensões da saúde positiva individual, porém a estratégia inicial permaneceu substantivamente inalterada.

Portanto, para medir diretamente o grau de saúde dos indivíduos, de modo similar aos procedimentos de screening para diagnóstico de doenças, têm-se desenvolvido e testado instrumentos estruturados e simplificados, capazes de reconhecer os estados de "completo bem-estar físico, mental e social" dos sujeitos. Digna de nota é a disponibilidade atual de escalas e inventários com essa finalidade, apresentando as mais diversas características metodológicas. Apenas para dar uma idéia dessa extraordinária proliferação, os primeiros guias sistemáticos desses instrumentos já listavam 71 diferentes tipos de escalas e questionários para a medida do estado individual de saúde, desde os pioneiros CMI (Cornell Medical Index) e GHQ (General Health Questionnaire), desenvolvidos respectivamente nas décadas de 60 e 70, até os contemporâneos EuroQol e QWBS (Quality of Well-Being Scale). ${ }^{29-30}$

Conforme tive a pretensão de analisar em A Clínica e a Epidemiologia,$^{10} \mathrm{o}$ aporte clínico contribui para a abordagem epidemiológica com critérios e operações de identificação de caso, determinando quem é e quem não é portador de uma dada patologia ou espécime de uma certa condição, na amostra ou na população estudada. Por esse motivo, o conceito de Risco constitui uma aproximação de segunda ordem do fenômeno da doença em populações, em última instância mediada pela Clínica como definidora da heterogeneidade primária do subconjunto [doentes].

Há um consenso em relação à centralidade da noção de 'doença' para o discurso científico e práxico da Clínica. ${ }^{12-22,31-34}$ Clavreul (1983) $)^{34}$ chega a apontar uma in- 
capacidade heurística da Clínica em definir os estados fisiológicos de saúde, salvo como ausência ou negação de doença. Ora, se a Clínica desenvolve-se como saber justificado pela noção de patologia, incapaz de reconhecer positivamente a presença ou ocorrência da saúde nos sujeitos individuais, pouco poderá fazer para colaborar na constituição de uma "epidemiologia da saúde”.* Dessa maneira, o fracasso da Clínica em subsidiar medidas positivas de saúde individual em princípio inviabilizaria a definição da heterogeneidade primária do subconjunto [sadios], imprescindível para qualquer abordagem epidemiológica da saúde coletiva, caso definida de modo rigoroso.

À guisa de balanço crítico dessa vertente, gostaria de assinalar os seguintes pontos:

(a) No nível individual, a Saúde não é um análogo inverso da doença. Se, para cada doença, observa-se um modo prototípico de adoecer (cujo reconhecimento implica uma semiologia clínica), há infinitos modos de vida com saúde, tantos quantos seres sadios.

(b) Ainda está por se estabelecer a validade conceitual dos construtos tomados como proxy da Saúde. A persistir tal lacuna, a investigação do desempenho operacional dos instrumentos correspondentes sempre encontrará dificuldades metodológicas sérias, principalmente em relação ao desenho de estudos de validade.

\section{A miséria da epidemiologia da saúde}

No nível coletivo, com menos propriedade ainda se pode falar em uma definição negativa de saúde. Podemos declarar (com algum esforço retórico) que um certo indivíduo é sadio porque nele não encontramos sinais de doença ou que um dado tipo de comportamento é saudável, na medida em que não se constitui em fator de risco para alguma enfermidade. Mas o que seria uma família sadia ou uma cidade saudável? Certamente que, ao indicar exemplares de uma ou outra condição, não estaremos falando de um grupo familial formado por indivíduos livres de enfermidade ou de uma comunidade em que ninguém morre ou adoece.

Como preliminar, gostaria de propor que o máximo de aproximação que a ciência epidemiológica tem se permitido consiste em definir Saúde como atributo do grupo de não-doentes, entre os expostos e os não-expostos a fatores de risco, em uma população definida. Na prática, a maioria dos manuais epidemiológicos é até bem menos sutil, chegando-se a definir a saúde diretamente como "ausência de doença". $\mathrm{Na}$ mesma medida em que o contingente de acometidos por uma dada patologia constitui o subconjunto populacional de referência para o cálculo do Risco, a 'saúde epidemiológica' implicaria por conseguinte meramente o contradomínio desse subconjunto:

$$
\text { Saúde }=(1-\text { Risco })
$$

Para melhor compreender essa questão, é preciso também discutir o fenômeno da comorbidade. O termo 'comorbidade' tem sido usado na Clínica para designar a existência concomitante de diferentes condições patológicas em um mesmo indivíduo. ${ }^{35}$ No âmbito epidemiológico, é bastante conhecido o processo equivalente de clustering de riscos em certos sujeitos e grupos populacionais, quando a presença de uma dada patologia aumenta a probabilidade de ocorrência de outras doenças naquele grupo suscetível. ${ }^{36}$

Não obstante as evidências em favor da complexidade das situações de saúde, os estudos epidemiológicos normalmente cobrem doenças específicas, buscando levantar o perfil sócio-demográfico dos expostos e dos doentes de uma dada patologia mais do que propriamente descrever o "perfil

* O que não impede a Clínica de usar e abusar da noção de "qualidade de vida" na retórica que sustenta sua prática cotidiana (devo essa observação a Jairnilson Paim, comunicação pessoal). 
patológico" (repertório de doenças e de condições relacionadas à saúde) de um dado grupo social. A soma de todos os casos de todas as doenças aparentemente não interessa muito à investigação epidemiológica. É quase irônico constatar que somente nesse caso seria possível visualizar uma verdadeira (porém trivial) definição negativa de Saúde, da seguinte maneira:

$$
\text { Saúde }=(1-\Sigma \text { Riscos })
$$

Para a estimativa de indicadores de níveis coletivos de saúde, no sentido positivo do construto, será imperativo superar uma limitação primordial da abordagem epidemiológica, originalmente restrita à avaliação dos riscos de doença ou de agravos. Assim, deve-se aperfeiçoar a sua capacidade de estimar medidas do grau de "morbidade negativa” ou de mensurar saúde como um análogo econométrico. Estamos falando aqui de duas estratégias distintas. Trata-se, no primeiro caso, de desenvolver metodologias e tecnologias capazes de avaliar positivamente os níveis de salubridade em uma dada população. No segundo caso, pretende-se desenvolver metodologias e técnicas para abordar a saúde enquanto o inverso do "volume global de patologia".

Analisemos brevemente a primeira dessas estratégias.

As técnicas de avaliação da saúde individual podem ser empregadas como fontes de elementos para a mensuração dos níveis coletivos de saúde tomados como somatório dos estados individuais de saúde. $\mathrm{Na}$ sua prática de produção de informação, a Epidemiologia tem instrumentalizado um repertório de "indicadores de saúde" que se baseia na contagem de doentes (indicadores de morbidade) ou de falecidos (indicadores de mortalidade). Propõe-se então, nesse caso, incluir entre as estratégias da Epidemiologia a contagem de indivíduos sadios, para isso desenvolvendo ou adaptando tecnologias pertinentes, no sentido analisado na seção anterior. Disso poderá resultar a derivação de indicadores de "salubridade”, equivalentes aos clássicos indi- cadores de morbidade. ${ }^{37}$ Nesse caso, contar-se-iam sadios para o cálculo de um certo risco de saúde, do mesmo modo como se computam doentes ou óbitos para a produção de indicadores de risco de doenças ou de mortalidade. Tal estratégia efetivamente não tem sido enfatizada no campo da investigação epidemiológica, limitandose a poucas avaliações de inquéritos domiciliares locais ou nacionais.

Para resumir um ponto de vista crítico em relação a essa estratégia de medida da saúde coletiva, gostaria de considerar o seguinte:

(a) Deve-se questionar se haverá fundamentação lógica na aplicação em nível agregado de construtos supostos como expressão da saúde de sujeitos individuais (função, desempenho, qualidade de vida, satisfação, bem-estar, felicidade etc.).

(b) Mesmo considerando a hipótese de uma demonstração convincente da validade dessa transposição, é lícito supor que a saúde coletiva significará sempre mais do que a somatória das saúdes individuais.

(c) A idéia de "risco de saúde" não é simétrica em relação à noção de risco de doenças porque, dentro do chamado raciocínio epidemiológico, as doenças são tomadas como eventos ou episódios, mensuráveis por meio de probabilidades condicionais de ocorrência.

Na segunda estratégia para a medida da saúde coletiva, propõe-se o desenvolvimento de medidas do "capital sanitário" ou da "carga de doença" de populações ou sociedades. As seções seguintes analisam mais detidamente esta alternativa.

\section{Uma pequena ajuda da economia da saúde}

Apesar das promessas de uma certa "epidemiologia da saúde", ${ }^{38}$ que chegou a inspirar a proposta de uma "sanometria", 39 dentre os indicadores ditos de saúde, apenas a medida denominada "Esperança de Vida” (às vezes equivocadamente designa- 
da por vida-média) e seus sucedâneos suportam uma definição não-residual de saúde. Mesmo listados nos manuais competentes da ciência epidemiológica, trata-se de indicadores mais demográficos que epidemiológicos, ainda assim também calculados com base em dados de mortalidade. Abordam anos de vida vividos, em geral sem considerar o estado ou nível de saúde desses anos ou, para incluir um conceito em moda atualmente, sem nada referir sobre a qualidade de vida dos sujeitos.

Na década de 60, Linder já defendia a necessidade de indicadores de saúde equivalentes ao GNP (gross national product), propondo desenvolver uma medida que denominou de GNHI (Gross National Health Index).$^{40}$ Sanders $(1964)^{41}$ havia anteriormente elaborado uma fórmula matemática de ajuste para a capacidade funcional de estimativas de vida-média, resultando em uma medida combinada de "anosde-vida-efetiva”, que foi aperfeiçoada por Moriyama (1968), ${ }^{42}$ introduzindo técnicas de análise de tábuas de vida. Sullivan (1971), ${ }^{43}$ com dados do censo e do National Health Interview Survey, pela primeira vez aplicou-a para computar medidas de expectativa de vida na ausência de incapacidades ou limitações. O aperfeiçoamento desta abordagem, incorporando distintas medidas de incapacitação, permitiu o desenvolvimento de toda uma família de indicadores de saúde geral, como por exemplo o YHL (Years of Healthy Life), usado nos Estados Unidos. ${ }^{44}$

No contexto de um ambicioso programa de avaliação tecnológica destinado a orientar as políticas de alocação de recursos para o NHS (National Health Service) da Inglaterra, uma equipe de pesquisa em Economia da Saúde da Universidade de York, sob a liderança de Alan Williams, ${ }^{45-47}$ desenvolveu o conceito de "qualidade de vida ligada à saúde" (health-related quality of life), assumidamente vinculado a uma teoria utilitarista da saúde. A abordagem metodológica correspondente resultou em um indicador da saúde positiva denominado QALY (quality-adjusted life years), esti- mado a partir do cálculo acumulado (por área geográfica ou divisão geopolítica) dos anos com qualidade de vida não-vividos por motivo de doença, incapacidade ou morte. Para as estimativas correspondentes, foi preciso classificar e ponderar distintas combinações de níveis de desconforto (distress) e incapacitação (disability), através de um intrumento de avaliação aplicado a "julgadores” da comunidade. A dimensão 'desconforto' divide-se em quatro níveis, variando de "nenhum desconforto" a "desconforto severo", enquanto que a dimensão “incapacitação" classifica oito estágios, desde plena capacidade até "inconsciência".

Com base nesses parâmetros, podia-se estimar o excedente de qalys produzidos por uma dada intervenção de saúde comparativamente em relação a outra tecnologia ou à ausência de intervenção. Assim, a efetividade de procedimentos destinados a restabelecer níveis satisfatórios de saúde com qualidade de vida poderiam ser avaliados com maior precisão, empregando-se a unidade de medida proposta. Um qaly significa um ano em perfeita saúde (nenhum desconforto; plena capacidade) porém pode também corresponder a dois anos com 0,50 qalyou quatro anos com 0,25 qalydo desempenho potencial do sujeito (equivalente a "saúde", caso aceitemos uma definição utilitarista deste conceito). Várias investigações nessa linha têm avaliado o impacto de tecnologias médicas, ${ }^{48}$ com fascinantes resultados: por exemplo, um transplante cardíaco em média cria 4,5 qalys, pouco menos do que uma série de hemodiálises (5 qalys) e equivalente a uma cirurgia de reconstituição de quadril (4,5 qalys). No entanto, o custo médio de um transplante é muitas vezes maior do que toda uma série de hemodiálises necessárias no período de sobrevida, já computados os ganhos correspondentes na qualidade de vida.

Sem dúvida, trata-se de uma proposição bem fundamentada teoricamente, considerando uma forma de definição positiva de saúde qualitativamente distanciada de qualquer concepção negativa da saúde referida à noção de patologia. $\mathrm{O}$ próprio 
Williams (1996, p. 1801) ${ }^{47}$ explicita o problema em um texto recente:

"Um tópico fundamental para esclarecer o que está em questão é o que se entende por 'saúde'. No contexto do QALY, trata-se de qualquer aspecto relativo à qualidade de vida que seja valioso para as pessoas, além da duração daquela vida. Isso deve ser claramente diferenciado de uma estrita definição clínica de saúde [...]"

O conceito do QALY, interessante sem dúvida, revela-se potencialmente útil para os seus objetivos primários de incorporar maior rigor e sofisticação às análises de custo-efetividade. Além disso, a sua concepção propiciou um importante desenvolvimento na teoria da mensuração em saúde, dadas as grandes possibilidades do seu emprego para medidas positivas da saúde individual como capacidade vital e qualidade de vida, de certo modo aí reduzidas a uma "unidade monetária" de troca, comparação e avaliação do valor diferencial de procedimentos restauradores ou promotores de saúde.

A abordagem do QALY tem se mostrado vulnerável a importantes críticas, de base política, sociológica, antropológica e ética, ${ }^{46-49}$ que não temos condição de aprofundar agora. De todo modo, frente à complexidade do processo de estabelecimento dos seus critérios e parâmetros, e considerando o caráter qualitativo e quase idiossincrático do construto qualidade-devida-relativa-a-saúde, deve-se reconhecer as dificuldades para o seu emprego em larga escala.

Tais restrições se aplicam especialmente a contextos sanitários com reduzido grau de desenvolvimento político e institucional, caracterizados por precários sistemas de informação em saúde. Justamente esses contextos são os que mais sofrem os efeitos das diversidades étnico-culturais e das desigualdades sociais. Em outras palavras, os conceitos de valor, utilidade, desconforto, incapacidade, qualidade de vida apresentam-se tão "ligados à cultura" e socialmente determinados que se pode questionar a validade teórica e o potencial comparativo de estratégias de medida da saúde como a abordagem do QALY.

\section{DALY: carga de doença ou estoque de saúde?}

Em 1992, no processo de preparação do 1993 World Development Report: Investing in Health, ${ }^{50} \mathrm{o}$ Banco Mundial contratou uma equipe da Escola de Saúde Pública da Universidade de Harvard, coordenada pelo economista Christopher Murray, para viabilizar uma metodologia destinada a medir a "carga global de doença" (GBD global burden of disease) das populações. Como pré-requisito fundamental, estabeleceu-se que os componentes de morbidade e mortalidade deveriam estar integrados em um mesmo indicador. Essa metodologia deveria ser também capaz de empregar dados epidemiológicos e estatísticas vitais em geral disponíveis, mesmo em países ditos subdesenvolvidos, de modo a permitir comparações internacionais, além de possibilitar avaliações do impacto dos investimentos internacionais e das políticas e programas de saúde. Ostensivamente inspirado no conceito do QALY, o novo indicador foi batizado de DALY (Disability-Adjusted Life Years) e definido como uma medida "do tempo vivido com incapacidade e do tempo perdido devido à mortalidade prematura". ${ }^{51}$

Parece-me apropriado, para os objetivos do presente trabalho, revisar em mais detalhe alguns aspectos metodológicos (inclusive matemáticos) do conceito de DALY que, nos termos dos seus formuladores, ${ }^{50-54}$ representaria tanto uma medida de carga de doença (morbidade e mortalidade) quanto um indicador de saúde mais transparente do ponto de vista ético. Esse destaque justificase, por um lado, pela enorme influência que tal proposta vem exercendo no panorama atual da política de saúde, em todo o mundo. Por outro lado, a proposta do DALY representa a mais importante tentativa recente de avançar a metodologia epidemiológica para superar o conceito de risco e seus correlatos. Em minha opinião, o debate em 
torno do DALY constitui a principal controvérsia epidemiológica dos anos 90 , talvez equivalente aos debates em torno da causalidade nos anos $70^{19} \mathrm{e}$ à polêmica sobre a epidemiologia clínica na década de $80{ }^{10}$

O DALY constitui um indicador composto na medida em que combina dados de mortalidade (anos de vida perdidos por óbito precoce) com dados de morbidade (grau e tempo de incapacidade devido a uma dada patologia). ${ }^{51-54}$ Estimam-se os anos de vida perdidos devido à mortalidade precoce tomando como padrão as expectativas de vida média de 80 anos para homens e 82,5 anos para mulheres. O tempo vivido sob incapacidade é calculado por meio de um conjunto de ponderações que supostamente refletem uma redução na capacidade funcional, por sua vez resultante de estudos de carga-de-doença específicos para cada morbidade. Para cada óbito ou caso registrado, computam-se os dalys correspondentes a serem acumulados para a estimativa das cargas-de-doença referentes a patologias específicas ou a agregados geopolíticos, como regiões, países ou continentes. $^{51-52}$

A principal novidade da proposta do DALY consistia na integração dos indicadores AVI - "anos vividos com incapacidade (YLD - years lived with disability) e AVP "anos de vida perdidos" (YLL - years of life lost) em uma única medida de "carga de doença”. ${ }^{53-54} \mathrm{O}$ conceito de 'incapacidade' passa a ser portanto crucial para o novo in- dicador proposto. Recuperando o modelo de progressão linear (doença, patologia, manifestação, deficiência, incapacidade, desvantagem) da International Classification of Impairments, Disabilities and Handicaps, ${ }^{55} \mathrm{o}$ conceito do DALY baseia-se na definição de incapacidade com "impacto da deficiência sobre o desempenho individual". ${ }^{53}$

Na sua proposta original, ${ }^{50}$ o componente incapacidade do DALY cobria quatro domínios da vida individual (procriação, ocupação, educação e recreação) e seis graus de severidade. As avaliações de grau de incapacidade por patologias selecionadas como "marcadores" eram realizadas por grupos de consenso de experts (primeiro, alunos internacionais de Harvard e depois, profissionais de saúde). Posteriormente, após avaliações por novos grupos de consenso internacionais, empregando-se o método do "person trade-off", ampliou-se a definição para "seqüelas incapacitantes de qualquer natureza” e acrescentou-se mais um grau de severidade da incapacitação. ${ }^{54} \mathrm{O}$ esquema resultante para a avaliação da incapacidade pode ser apreciado na Tabela.

Em sua expressão mais direta, algebricamente a fórmula do DALY é intrigantemente simples:

$$
\mathrm{DALY}_{i}=\mathrm{AVP}+\mathrm{AVI}
$$

sendo $\mathrm{AVP}=$ anos de vida perdidos, $\mathrm{AVI}=$

Tabela - Graus de severidade, pesos e condições-índice de incapacidade do projeto GBD.

Table - Degrees of severity, weights and conditions - the GBD project incapacity rate

\begin{tabular}{ccl}
\hline Grau & Peso & Condição-índice \\
\hline 1 & $0,00-0,02$ & Vitiligo, Baixo Peso \\
2 & $0,02-0,12$ & Diarréia, Anemia Severa \\
3 & $0,12-0,24$ & Infertilidade, Artrite, Angina \\
4 & $0,24-0,36$ & Amputação de Membro Inferior, Surdez \\
5 & $0,36-0,50$ & Fístula Retovaginal, Retardo Mental Moderado \\
6 & $0,50-0,70$ & Depressão Unipolar, Cegueira, Paraplegia \\
7 & $0,70-1,00$ & Psicose Grave, Demência, Quadriplegia \\
\hline
\end{tabular}

Fonte: Adaptado de Murray e Acharya ${ }^{54} 1997$.

Source: Adapted from Murray \& Acharya ${ }^{54}, 1997$. 
anos vividos com incapacidades, tomando $i$ como a designação da patologia ou grupo de morbidade para o qual se computam os casos ou óbitos.

Entretanto, visando reforçar as propriedades econométricas do indicador, os formuladores do DALY decidiram incorporar dois tipos de função de correção: a) um desconto para anos ainda não vividos, equivalente à noção de taxa de juros para pagamentos futuros; b) uma ponderação por idade, destinada a corrigir o valor dos anos vividos em relação a cada etapa do ciclo de vida." Pode-se assim derivar para cada AVP ou AVI diferentes alternativas de correção.

Para o cálculo do componente AVP do DALY, na sua formulação mais simplificada, emprega-se a seguinte equação:

$$
\operatorname{AVP}[0,0]=\mathrm{V}_{a}
$$

onde $\mathrm{V}$ equivale diretamente à expectativa de vida na idade $a$ (calculada em relação às expectativas de vida médias de 80 e 82,5 anos, respectivamente para homens e mulheres), sem desconto nem correção.

Com a ponderação por idade, aplicando-se a função de correção C, temos

$$
\begin{array}{r}
\operatorname{AVP}[0, k]=\left(k C_{e}^{-\beta a} / \beta 2\right) \mathrm{e}^{-\beta V}[-\beta(\mathrm{V}+a)-1] \\
-[-\beta-1]+[\mathrm{V}(1-k)]
\end{array}
$$

Finalmente, aplicando-se uma taxa de desconto $d \neq 0$, a fórmula completa desse componente do DALY é a seguinte:

AVP $[d, k]=\left[\mathrm{kC}_{\mathrm{e}}{ }^{d a} /(d+\beta)^{2} \mathrm{e}^{-(d+\beta)(V+a)}\right.$ $[-(d+\beta)(\mathrm{V}+a)-1]-[-(d+\beta) a-1] \mathrm{e}^{-(d+\beta) a}$ $+\left(1-\mathrm{e}^{-d V}\right)[(1-k) / d]$

Para o cálculo do componente AVI do DALY, por sua vez, a expressão mais simples corresponde à seguinte fórmula:

$$
\text { AVI }[0,0]=I\left(D_{0,0}\right)
$$

onde $\mathrm{I}$ = peso relativo da incapacidade $\mathrm{e}$
$\mathrm{D}_{0,0}=$ duração da incapacidade sem correções nem descontos. De modo análogo ao cálculo dos AVP, apresentado acima, podemos estimar os AVI com correção por idade e, além disso, com desconto por anos vividos sem incapacidade no futuro.

No primeiro caso, temos:

$$
\text { AVI }[0, k]=\mathrm{I}\left(\mathrm{D}_{0, k}\right)
$$

onde $\mathrm{D}_{0, k}$ equivale à duração da incapacidade corrigida por idade, na condição de $d=0$, calculada de modo equivalente a [2.1] acima.

No segundo caso, temos:

$$
\mathrm{AVI}[d, k]=\mathrm{I}\left(\mathrm{D}_{d, k}\right)
$$

sendo que $\mathrm{D}_{d, k}$ indica duração média da incapacidade corrigida por idade e com desconto por anos com incapacidade vividos no futuro $(d \neq 0)$, calculada de maneira similar a [2.2] acima.

Não obstante o suporte ideológico, político e financeiro que uma instituição do peso do Banco Mundial vem concedendo a essa proposição, contra ela avolumam-se críticas teóricas e metodológicas, principalmente em relação aos seguintes aspectos:

(a) Reduz a saúde a perfis de doenças, com indicadores unidimensionais da situação de saúde, sabidamente complexa e multifacetada, ao tempo em que negligenciam elementos não-quantitativos essenciais para a determinação dos níveis de saúde. ${ }^{56}$

(b) Condensa medidas de mortalidade e de morbidade com graus heterogêneos de precisão, resultando em uma acurácia ilusória. ${ }^{49,56-57}$ Os indicadores assim produzidos padecem de excessivo nível de agregação, escamoteando importantes desigualdades em saúde, principalmente segundo condições de vida. ${ }^{58}$

(c) Baseia-se em um número excessivo de pressupostos e ajustes arbitrários (pesos, descontos e correções) que comprometem a objetividade da medida,

* A ponderação por idade é obtida através da função de correção $k=C_{x e}{ }^{-\beta x}$, onde $x=$ idade, $\beta=0,04$ e $C=0,16243$ constituem constantes de padronização para cada grupo etário. ${ }^{54}$ 
distanciando-a das realidades concretas de saúde que supostamente constituem seu objeto privilegiado. ${ }^{56-58}$

Avaliando globalmente a questão, Barker e Greene ${ }^{57}$ comentam que essa proposta termina forçando um recuo ao modelo biomédico de cuidado à saúde. Porém as críticas mais comprometedoras do projeto metodológico do consórcio Banco Mundial - Universidade de Harvard dizem respeito aos aspectos éticos e políticos do sistema de financiamento e tomada de decisão implícitos no Projeto GBD . ${ }^{58-61}$ Algumas dessas críticas surgiram de dentro do próprio Banco Mundial (Hammer, 1999, p. 8):61

"Vários dos pressupostos para cálculo do DALY podem bem violar julgamentos éticos. [...] Em particular, simplesmente somar dalys em indivíduos implica tratar a perda da vida inteira de uma pessoa como a mesma coisa que a perda de um dia de cada um. [...] Se isto faz sentido ou não, não é um problema matemático dado que as suas implicações são debitadas inteiramente às leis da adição. [...] Temos bons motivos para lidar com dinheiro dessa maneira porém a extensão desse método para comparar as vidas de indivíduos que vivem em momentos diferentes é obviamente mais problemático."

Enfim, questiona-se se efetivamente a metodologia DALY atinge os objetivos propostos, na medida em que promete algo bastante mais complexo do que estimar impacto de políticas ou programas de saúde, como por exemplo tornar-se a base técnica de toda uma revolução no conceito de política de saúde, designado pomposamente como "evidence-based health policy", 62-63 através da avaliação da "carga global de doença” de uma dada sociedade. O Subcommittee on Health Measurement da Organização Mundial da Saúde liminarmente já indicava que, em geral, os indicadores de base econométrica como o DALY na verdade "falham em refletir a carga de doença em um país ou em uma comunidade" ${ }^{56}$

Os formuladores do DALY têm se empenhado em considerar essas críticas, ab- sorvendo-as no aperfeiçoamento da proposta ou buscando rebatê-las, nesse caso com reduzido sucesso. Murray e Lopez ${ }^{52}$ apresentaram em 1996 um exercício de aplicação do método DALY que incorporava maior padronização para os diferentes componentes da medida. De certa forma respondendo ao ponto (b) acima, Murray $(1996)^{53}$ argumenta ainda que, mais que medir iniqüidades em saúde, deve-se desenvolver e aplicar estratégias de promoção da eqüidade, para o que se necessita de medidas como o DALY. Considerando a crítica (a), Murray e Acharya (1997) ${ }^{54}$ defendem que um DALY contextualizado e multifacetado seria "indesejável e inviável", não atendendo aos requisitos de robustez e comparabilidade exigidos de um indicador dessa natureza. Os mesmos autores, incapazes de rebater todos os argumentos de Anand e Hanson (1997), ${ }^{58}$ sem dúvida os críticos da proposta DALY de maior densidade metodológica, recomendam que os planejadores e tomadores de decisão que duvidarem dos pressupostos do método usem medidas sem ajustes e sem correções, em vez das versões corrigidas (respectivamente DALY[0,0], DALY $[0, k]$ e DALY $[d, k]$, como vimos acima nesta mesma seção).

Em minha opinião, é preciso considerar que, por um lado, em comparação com os seus antecessores da linha QALY, o DALY realmente representa uma simplificação no sentido de que opera com uma única dimensão de medida individual de saúde, o nível de comprometimento funcional, em lugar de uma escala subjetiva de valores combinados de desconforto e incapacidade. ${ }^{57}$ Por outro lado, implica também uma ampliação de escopo da metodologia proposta, na medida em que se apresenta sem hesitação como um quantificador macroeconômico de "volumetria" da morbi-mortalidade. $^{54}$

Sejamos pelo menos justos com os objetivos dos criadores e promotores dessa abordagem. O termo "carga global de doença" é bastante claro e preciso no sentido de definir a doença e não a Saúde como objeto: de modo algum induz a falsas pro- 
messas. Esse aspecto é conscienciosamente assinalado pelos formuladores do conceito DALY (Murray e Acharya, 1997, p. 705), ${ }^{54}$ da seguinte maneira:

"Não obstante, deve-se notar que em geral um QALY implica um conceito positivo, um análogo em relação a um ano de vida saudável. Um DALY é um conceito negativo, um ano de vida saudável perdido. (Itálicos meus)"

Podemos concordar que QALY e DALY de facto constituem medidas globais de morbi-mortalidade e não indicadores do estoque de saúde de uma dada sociedade. Curiosamente, ambas as abordagens utilizam anos vividos com qualidade de vida ou sem incapacidade (que é um índice grosseiro de saúde) para avaliar o impacto social de patologias e das tecnologias destinadas à sua prevenção, controle ou erradicação. Trata-se de uma aplicação da esquisita noção de doença = ausência de saúde, invertida da concepção convencional de saúde como ausência de doença.

\section{Conclusão: da Quantofrenia à Sanologia?}

Aparentemente esgotam-se os argumentos que validam heurística e eticamente o repertório das propostas de abordagens metodológicas destinadas à avaliação direta dos níveis coletivos de saúde através da panacéia dos indicadores unificados, superando uma fase que, nos seus primórdios, foi astutamente designada por Harald Hansluwka como "the other extreme of quantophrenia" ${ }^{64}$ Devemos, portanto, demandar da abordagem econométrica da saúde o que ela tem de melhor a oferecer, principalmente nas áreas de avaliação tecnológica e microeconomia em saúde. Isto significa aceitar as suas limitações enquanto instrumento de mensuração do grau de "salubridade" global de uma dada população.

Iniciamos o nosso percurso avaliando os resultados de esforços no sentido de aperfeiçoar formas de mensuração de uma certa "síndrome da saúde", como se viável fos- se o projeto de lidar com Saúde enquanto mera ausência de doença. Abordamos criticamente a proposta teórica e metodológica de uma "sanometria", ${ }^{39}$ apontando seus impasses e limites. Concluímos que as proposições mais atuais e aparentemente mais sofisticadas, como as estimativas da "qualidade de vida relativa a saúde" e a abordagem do GBD, não passam de medidas da doença e seus efeitos (incapacidade e mortalidade).

Creio que talvez seja possível, ainda, cultivar alguma expectativa em relação aos conceitos correlatos de HALE (health-adjusted life expectancy), ora em desenvolvimento no Canadá, ${ }^{65}$ e DFLE (disability-free life expectancy), aperfeiçoado e adotado na França ${ }^{66}$ Constituem, esses sim, "verdadeiros" indicadores de vida vivida com saúde, pelo menos no sentido de que descartam períodos vividos com doença ou incapacidade para tentar estimar uma expectativa média de anos de vida saudável para populações ou grupos sociais. Em qualquer dos casos, o desenvolvimento teórico e principalmente metodológico necessário para formalizar e aplicar essas propostas na análise de situações concretas de saúde ainda se mostra insuficiente. Mas pelo menos se pode reconhecer um movimento no sentido de definir pragmaticamente (ou quiçá trivialmente, diria um crítico mais rigoroso) saúde como vida com saúde, ou seja, anos vividos com funcionalidade produtiva e social. ${ }^{67}$

Não obstante os problemas e limitações apontados, todo esse esforço representa uma incalculável contribuição no sentido de avançar o processo teórico da saúde. Temos que considerar o potencial heurístico acumulado nas interfaces entre as ciências sociais e as ciências da saúde. Penso que aí será possível encontrar algumas condições objetivas para a formulação de uma proposta de, primeiro, sistematização e, em seguida, de meta-síntese do problema científico da Saúde.

Assim, a despeito das boas intenções, sinceros esforços e denodados manifestos em prol de uma "epidemiologia da saúde", ${ }^{38,68}$ aparentemente resta à Epide- 
miologia a alternativa de abandonar o conceito clássico de Risco e construir um novo objeto-modelo. Para remover o incômodo ponto-cego, objeto deste ensaio, a disciplina terá que realizar um imenso investimento de formulação teórica. Nesta etapa ainda preliminar, será necessário produzir esquemas explicativos meta-sintéticos, ancorados na realidade concreta de saúde.

Não tenho dúvidas de que o objeto possível da saúde-doença-cuidado é um desses objetos heurísticos complexos, plurais e sensíveis aos contextos, que só se define em sua configuração total, já que a apreensão de cada um dos seus elementos e dimensões não nos dá acesso à integralidade deste objeto. ${ }^{69}$ Tenho dúvidas, isso sim, de se a Epidemiologia, tal como a conhecemos, terá condições de enfrentar esse desafio. Se nosotros epidemiologistas quisermos e se os nossos outros deixarem, poderemos reformá-la, reforçá-la, revolucioná-la. Mas será este esforço suficiente para fazer a nos- sa ciência enfim enxergar o seu ponto-cego? Ou talvez, num processo de construção histórica, será mesmo necessário inaugurar uma nova ciência para esse novo/velho objeto complexo? Poderíamos, quem sabe, batizá-la de Sanologia.

Em conclusão, face ao fracasso do projeto de uma "epidemiologia da saúde", pelo menos em bases simétricas à "epidemiologia dos riscos" que conhecemos, e frente às limitações da nova geração de indicadores ditos de saúde, o desafio mais crucial no processo de aggiornamento da Epidemiologia será justamente a questão do objeto Saúde. No presente momento, é preciso urgente avançar para um tratamento epistemológico e teórico deste intrigante ponto cego, objeto-modelo potencial para uma nova definição da Saúde em sociedades concretas, buscando produzir reflexões e indicações conceituais e metodológicas capazes de enriquecer a pesquisa e a prática no campo da Saúde Coletiva.

\section{Summary}

In this paper, the author evaluates the proposition that the concept of 'Health' is a blind-spot for epidemiologic science. Initially, he discusses the limitations of the conventional epidemiologic approach, linked to a clinical definition of disease, in order to build its object: Health. He concludes that there is no logical basis for a negative definition of Health, neither at the individual level nor at the collective level. Secondly, the author briefly analyzes the attempts of producing an epidemiology of health symmetric to the epidemiology of risks, defending the feasibility of counting healthy subjects for calculating "risk of health" in the same way as diseased or deceased are computed for producing risk indicators of diseases or mortality. Thirdly, he critically evaluates the econometric approaches that reinforce and complement the epidemiologic repertoire conceived for health measurement, such as the QALY method. Concepts that are related to Health such as value, utility, well-being, disability, and quality of life are so "culture-bound" and socially determined that the theoretical validity and the comparative potential of such strategies for measuring health are questioned. Due to its growing importance in the definition of budgetary policies in health, the author focuses in more detail on the so-called DALY approach, sponsored by the World Bank and WHO, considered as a prototype of the new generation of health indicators. He discusses a series of theoretical and methodological criticisms that such a proposal will eventually become a retreat to a biomedical model of health care, in addition to those concerning the ethical and political aspects of the financing 
and decision-making systems implicit in the DALY. Finally, recognizing the failure of methodological proposals for a direct evaluation of levels of collective health through unified indicators, the author concludes with an evaluation of the current prospects for Epidemiology in order to integrate the complex object of Health into its theoretical and methodological agenda.

\section{Referências}

1. Arouca A S. O dilema preventivista: contribuição para a compreensão e crítica da Medicina Preventiva. [Tese de Doutoramento]. Campinas: UNICAMP; 1975.

2. Callahan D. The WHO definition of health. Stud Hastings Center 1973; 1(3).

3. Anderson R. Health promotion: an overview. Eur Monogr Health Educ Res 1984; 6:1-126.

4. Editorial. Am J Health Promotion 1989; 3:3-5.

5. Nutbeam D. Evaluating health promotion: progress, problems and solutions. Health Promot Int 1999; 13: 27 43.

6. OMS/UNICEF. Cuidados primários de saúde. Brasília (DF); 1979 [Relatório da Conferência Internacional sobre Cuidados Primários de Saúde; 1978, 6-12 set; Alma-Ata, URSS].

7. Lalonde M. A new perspective on the health of Canadians. Ottawa: Information Canada; 1974.

8. WRR Netherlands. Volksgezondheidszorg. The Hague, Scientific Council for Government Planning; 1997.

9. Paim J. A reforma sanitária e os modelos assistenciais. In: Rouquayrol MZ, Almeida Filho N. Epidemiologia e saúde. Rio de Janeiro: Medsi; 1999. p. 473-87.

10. Almeida Filho N. A clínica e a epidemiologia. 2a ed. Salvador. APCE/Rio de Janeiro: ABRASCO; 1997.

11. Almeida Filho N. The paradigm of complexity: applications in the field of public health. In: Advisory Committee on Health Research. A research policy agenda for science and technology to support global health development. Geneva: World Health Organization; 1997. p.1-15.

12. Temkin O. The scientific approach to disease: specific entity and individual sickness. In: Crombie A, editor. Scientific change: historical studies in the intellectual, social and technical conditions for scientific discovery. New York: Basic Books; 1963. p. 629-47.

13. Murphy E. The logic of medicine. Baltimore: Johns Hopkins University Press; 1965.

14. Reznek L. The nature of disease. London: Routledge \& Keegan Paul; 1987.

15. Pérez-Tamayo R. El concepto de enfermedad. Mexico (DF): Fondo de Cultura Económica; 1988.
16. Berlinguer G. A doença. São Paulo: CEBES/Hucitec; 1988.

17. Abed LC. La enfermedad en la história. Cordoba: Direccion General de Publicaciones, Universidad Nacional de Cordoba; 1993.

18. Kaufman F. Disease: definition and objectivity. In: Humber R, Almeder J, editors. What is disease. Totowa (NJ): Humana Press; 1997. p. 269-86.

19. Nordenfeld L, Odelstad J, editors. Health, disease, and causal explanations in medicine. Dordrecht: D. Reidel; 1984.

20. Humber JM, Almeder R editors. What is disease? Totowa (NJ): Humana Press; 1997.

21. Almeida Filho N. Epidemiologia sem números. Rio de Janeiro: Campus; 1989.

22. Canguilhem G. Le normal et le pathologique. Paris: Presses Universitaires de France; 1966.

23. Noack H. Concepts of health and health promotion. In: Abelin T, Brzezinski Z, Carstairs V, editors. Measurement in health promotion and protection. Copenhagen: WHO Regional Office for Europe; 1987. p. 5-28. (WHO Regional Publications, European Series, 22).

24. Patrick D, Erikson P. Health status and health policy: quality of life in health care. New York: Oxford University Press; 1993.

25. Robine JM. Measurement of states of health in populations: dimensions and levels. In: Sayers B. Health assessment - complexity, trends and opportunities. Geneva: WHO Global Advisory Committee for Health Research; 1999. (Appendix III).

26. Davies DF. Progress toward the assessment of health status. Prev Med 1977; 4: 282-95.

27. Kaplan B, Cassel J, Gore S. Social support and health. Med Care 1977; 15(5): 47-58.

28. Corin E. The social and cultural matrix of health and disease. In: Evans RG, Barer ML, Marmor R, editors. Why are some people healthy and others not? The determinants of health of populations. Hawthorn (NY): Aldine de Gruyter; 1995. p. 93-132.

29. Bowling A. Measuring health: a review of quality of life measurement scales. Buckingham: Open University Press; 1997. 
30. McDowell I, Newell C. Measuring health: a guide to rating scales and questionnaires. New York: Oxford University Press; 1987.

31. Foucault M. Naissance de la clinique: une archéologie du régard médical. Paris: P.U.F.; 1963.

32. Boorse C. On the distinction between disease and illness. Philosophy Public Affairs 1975; 5: 49-68.

33. Castiel L.D. O buraco e o avestruz: a singularidade do adoecer humano. Campinas: Papirus; 1994.

34. Clavreul J. A ordem médica. São Paulo: Brasiliense; 1980.

35. Crabtree HL, Hildreth AJ, O'Connell JE, Brown J. The Comorbidity Symptom Inventory: a combined inventory of disease and assessment of symptom severity. J Am Geriatric Assoc 1999; 49: 102-12.

36. Chen W, Srinivasan SR, Elkasabany A et al. Casdiovascular risk factors clustering features of insulin resistance syndrome in a biaracial population of children, adolescents and young adults. Am J Epidemiol 1999; 150: 667-74.

37. Uemura K. Application of indicators for monitoring progress towards health for all by the year 2000. In: Abelin T, Brzezinski Z, Carstairs V, editors. Measurement in health promotion and protection. Copenhagen: WHO Regional Office for Europe; 1987. p. 643-52.(WHORegional Publications, European Series, 22).

38. Galdston I, editor. The epidemiology of health. New York: Health Education Council; 1953.

39. Goldberg M, Dab W, Chaperon J, Fuhrer R, Gremy F. Indicateurs de santé et "sanométrie": les aspects conceptuels des recherches récentes sur la mesure de l'état de santé d'une population. Rev Epidémiol Santé Publique 1979; 27: 51-68.

40. Linder F. The health of the American people. Sci Am 1966; 214(6):21-9.

41. Sanders B. Measuring community health levels. Am J Public Health 1964; 54: 1063-70.

42. Moriyama I. Problems in the measurement of health status. In: Sheldon E, Moore W, editors. Indicators of social change. New York: Russel Sage Foundation; 1968. p. 573-600.

43. Sullivan DF. A single index of mortality and morbidity. HMSHA Health Rep 1971; 86: 347-55.

44. Erikson P, Wilson R, Shannon I. Years of healthy life. Healthy people 2000 statistical notes. Hyattsville: National Center for Health Statistics/CDC/DHHS; 1995.

45. Williams A. The nature, meaning and measurement of health and illness: an economic viewpoint. Soc Sci Med 1985; 20: 1023-7.
46. Williams A. The importance of quality of life in policy decisions. In: Walker S, Rosser R, editors. Quality of life: assessment and application. Dordrecht: MTP; 1993. p. 427-39.

47. Williams A. QALYs and ethics: a health economist's perspective. Soc Sci Med 1996; 43: 1795-804.

48. Loomes G, MacKenzie L. The use of QALYS in health care decision making. Soc Sci Med 1989; 28: 299-308.

49. Fryback D. Health-related quality of life or population health measures: a brief overview of the HALY Family of Measures. Washington (DC): National Academy of Sciences; 1997. [Workshop on Summary Measures of Population Health Status]

50. World Bank. World development report 1993: investing in health. New York: Oxford University Press; 1993.

51. Murray CL. Quantifying the burden of disease: the technical basis for disability-adjusted life years. Bull World Health Organ 1994; 72: 429-45.

52. Murray CL, Lopez A, editors. The global burden of disease. Cambridge: Harvard University Press; 1996. (Global Burden of Disease and Injuries Series, 1).

53. Murray CL. Rethinking DALYs. In: Murray CL, Lopez A, editors. The global burden of disease. Cambridge: Harvard University Press; 1996.

54. Murray CL, Acharya AK. Understanding DALYs. J Health Econ 1997; 16: 703-30.

55. World Health Organization. International Classification of Impairments, Disabilities and Handicaps. Geneva: WHO; 1980.

56. World Health Organization. Progress report - DALY Review Group. ACHR Subcommittee on Health Measurement. Geneva; 1996.

57. Barker C, Greene A. Opening the debate on DALYs. Health Policy Plann 1996; 11:179-83.

58. Anand S, Hanson K. Disability-adjusted life years: a critical review. J Health Econ 1997; 16:685-702.

59. Daniels N. Distributive justice and the use of summary measures of population health status. Washington (DC): National Academy of Sciences; 1997. [Workshop on Summary Measures of Population Health Status].

60. Brock D. Ethical issues in the development of summary measures of population health status. Washington (DC): National Academy of Sciences; 1997. [Workshop on Summary Measures of Population Health Status].

61. Hammer J. Health research and decision making. In: Sayers B. Health assessment: complexity, trends and opportunities. Geneva: WHO Global Advisory Committee for Health Research; 1999. (Appendix I). 
62. Murray CJL, Lopez AD. Evidence-based health policy: lessons from the Global Burden of Disease Study. Science 1996; 274(5288): 740-3.

63. Rada J, Rati M, Howden-Chapman P. Evidence-based purchasing of health promotion: methodology for reviewing evidence. Health Promot Int 1999; 14: 177-88.

64. Hansluwka H. Measuring the health of populations. Indicators and interpretations. Soc Sci Med 1985; 24: 1207-24.

65. Wolfson A. Health-adjusted life expectancy. Health Rep Stat Can 1996; 8:41-6.
66. Robine J-M. Romieu I, Cambois E. Health expectancy indicators. Bull World Health Organ 1999; 77: 181-5.

67. Nordenfeld L. On the nature of health. Dordrecht: D. Reidel Publications; 1987.

68. Terris M. La revolución epidemiológica y la medicina social. México (DF): Siglo XXI; 1980. p. 39-60.

69. Almeida Filho N. The complex object: health. In: Sayers B. Health assessment: complexity, trends and opportunities. Geneva: WHO Global Advisory Committee for Health Research; 1999. (Appendix II). 